\title{
CKM mixings from mass matrices with five texture zeros
}

\author{
Yithsbey Giraldo ** and Eduardo Rojas ${ }^{\dagger}$ \\ Departamento de Física, Universidad de Nariño, A.A. 1175, San Juan de Pasto, Colombia
}

(Received 19 May 2021; revised 15 September 2021; accepted 17 September 2021; published 7 October 2021)

\begin{abstract}
In this work we carry out an exhaustive study to find quark mass matrices in the Standard Model (SM), with the maximum number of texture zeros consistent with the experimental data. We found four viable configurations of five texture zeros that adjust the quark masses, the mixing angles and the $C P$ violation phase, with deviations below $1 \sigma$ level respect to the current SM best fit values. One of the most important aspects of this work is an economic procedure to find the texture zeros: we resort to the weak basis transformation method, which, as we will show, exhaustively search every possible configuration. We report various leading order relations between the mixing angles and the quark masses for each case.
\end{abstract}

DOI: 10.1103/PhysRevD.104.075009

\section{INTRODUCTION}

In the Standard Model (SM), the quark mass matrices come from the interaction between the Higgs boson and the SM fermions. After the spontaneous breaking of the SM gauge symmetry we obtain

$$
-\mathcal{L}_{M}=\bar{u}_{R} M_{u} u_{L}+\bar{d}_{R} M_{d} d_{L}+\text { H.c. },
$$

where $M_{u}$ and $M_{d}$ are arbitrary, $3 \times 3$ quark mass matrices containing thirty-six (36) real parameters, which cannot be fully determined from the ten (10) physical observables that they must account for: six (6) quark masses, three (3) flavor mixing angles, and one (1) charge-parity $(C P)$ violating phase. However, in models like the SM (or its extensions) where the right fields are singlets under the gauge group, it is always possible to choose a suitable basis for the right quarks, such that by using the polar decomposition theorem of the matrix algebra, the mass matrices of type "up" and "down" became Hermitian [1-6].

$$
M_{u}^{\dagger}=M_{u}, \quad \text { and } \quad M_{d}^{\dagger}=M_{d}
$$

Additionally, for Hermitian quark mass matrices, you can make a unitary transformation acting simultaneously on the up-type and down-type quark mass matrices, leaving the gauge currents invariant, and the mass matrices transform to new equivalent Hermitian matrices

\footnotetext{
yithsbey@gmail.com

†eduro4000@gmail.com
}

Published by the American Physical Society under the terms of the Creative Commons Attribution 4.0 International license. Further distribution of this work must maintain attribution to the author(s) and the published article's title, journal citation, and DOI. Funded by SCOAP ${ }^{3}$.
$M_{u} \rightarrow M_{u}^{\prime}=U^{\dagger} M_{u} U, \quad M_{d} \rightarrow M_{d}^{\prime}=U^{\dagger} M_{d} U$,

where $U$ is an arbitrary unitary matrix that preserves the hermiticity of the mass matrices and leaving the physical quantities invariant, in particular, the Cabibbo-KobayashiMaskawa (CKM) mixing matrix. This common unitary transformation applied to $M_{u}$ and $M_{d}$, in Eq. (1.3), is known as a "weak basis" (WB) transformation [1,7-10]. As it was shown in $[3,11]$, for a given set of quark masses, mixing angles and the $C P$-violating phase, all the mass matrices consistent with these experimental values are unitarily equivalent. This result can be used to calculate the maximum number of texture zeros, since it guarantees that by using WB transformations it is possible to reach all physical and nonphysical zeros consistent with the data $[3,7]$. Through a WB transformation, it is possible to rewrite the quark mass matrices as follows $[3,7,11,12]$ :

$$
\begin{aligned}
& M_{u}=D_{u}=\left(\begin{array}{ccc}
\lambda_{1 u} & 0 & 0 \\
0 & \lambda_{2 u} & 0 \\
0 & 0 & \lambda_{3 u}
\end{array}\right), \\
& M_{d}=V D_{d} V^{\dagger},
\end{aligned}
$$

or

$$
\begin{aligned}
& M_{u}=V^{\dagger} D_{u} V, \\
& M_{d}=D_{d}=\left(\begin{array}{ccc}
\lambda_{1 d} & 0 & 0 \\
0 & \lambda_{2 d} & 0 \\
0 & 0 & \lambda_{3 d}
\end{array}\right),
\end{aligned}
$$

where $V=U_{u}^{\dagger} U_{d}$ is the CKM mixing matrix, $U_{u}$ and $U_{d}$ are the diagonalization matrices for the mass matrices $M_{u}$ and $M_{d}$, respectively. The parameters $\lambda_{i q}(i=1,2,3)$ are the quark mass matrix eigenvalues for up-type $(q=u)$ and 
down-type $(q=d)$ quarks, which are related to the quark masses

$$
\begin{aligned}
\left|\lambda_{1 u}\right|=m_{u}, & \left|\lambda_{2 u}\right|=m_{c}, & \left|\lambda_{3 u}\right|=m_{t}, \\
\left|\lambda_{1 d}\right|=m_{d}, & \left|\lambda_{2 d}\right|=m_{s}, & \left|\lambda_{3 d}\right|=m_{b} .
\end{aligned}
$$

So $\lambda_{i q}$ can be positive or negative and obey the hierarchy

$$
\left|\lambda_{1 q}\right| \ll\left|\lambda_{2 q}\right| \ll\left|\lambda_{3 q}\right| .
$$

In the basis (1.4) can be easily verified that the mass matrices are consistent with the CKM mixing matrix $V$ and the quark masses, and the 3 nonphysical texture zeros can be effortlessly identified [7]. The hermiticity of the quark mass matrices $M_{u}$ and $M_{d}$ reduces the number of free parameters from 36 to 18 , which, however, is still a large value compared to the number of observables. In order to reduce the number of free parameters, Weinberg and Fritzsch [13-16] introduced texture-zeros into the mass matrices with a dual purpose, first of all, to obtain selfconsistent relationships between the quark masses and the flavor mixing parameters that can be experimentally verified $[1,17]$. On the other hand, the discrete (or continuous) flavor symmetries hidden in such textures may finally provide clues on the origin of the energy scales in the quark sector of the SM as residual symmetries of a more fundamental symmetry at high energies. Hermitian quark mass matrices with six texture zeros were introduced in what is currently known as the Fritzsch type [16,17], where the mass matrices, $M_{u}$, and $M_{d}$, have the same texture ("updown" parallel) each with three zeros. This type of ansatz was ruled out due to the large value of the mass of the top quark, since that for this case the CKM element $\left|V_{c b}\right|$ is in tension with the experimental data $[4,17,18]$. Furthermore, for reasonable values of the current quark masses $m_{u}$ and $m_{c}$, the expected magnitude for $\left|V_{u b} / V_{c b}\right|=\sqrt{m_{u} / m_{c}} \approx$ 0.05 [19] is too small in comparison with the experimental value $\left(\left|V_{u b} / V_{c b}\right|_{\text {exp }} \approx 0.09\right.$ [20-22]). In this sense, one of the difficulties of working with texture zeros is keeping the predictions for $V_{u s}$ and $V_{c d}$ right, and simultaneously reproducing the ratios $V_{u b} / V_{c b}$ and $V_{t d} / V_{t s}$, i.e.,

$$
\begin{aligned}
\left|\frac{V_{u b}}{V_{c b}}\right|_{\exp } & =0.0861 \pm 0.0027 \\
\left|\frac{V_{t d}}{V_{t s}}\right|_{\exp } & =0.2107 \pm 0.0044 .
\end{aligned}
$$

The original literature on five-zero textures has been widely studied, but these initial ansatzes are not currently favored by experimental data $[4,7,23-28]$. Recent studies show that other five-zero textures are viable, some analytical and numerical examples were reported in [3,5,29-31], these textures reproduce the quark masses and the CKM mixing matrix with deviations respect to the experimental values below $1 \sigma$ level. There are several approaches to obtain the texture zeros, in some cases, the analytic approximations take advantage of the strong hierarchy in quark masses and mixing angles to motivate a certain texture [17,32], alternatively, some techniques prefer to assume a texture for the quark mass matrices to make physical predictions $[4,29,31,33]$. A very elegant way is to apply WB transformations in order to get texture zeros in the mass matrices [3,7], our work points in this direction and it can be considered as a continuation of the work presented by one of us in [3]. This work is organized as follows: In Sec. II we classify all possible ways to put three texture-zeros in the "up" or "down" quark mass matrices. This analysis is important since from these textures we can obtain five texture zeros for the mass matrices by using the WB transformation method. We will carry out a first analytical study for five-zero textures in Sec. III, and the conclusions are summarized in Sec. IV.

\section{FIVE-ZERO TEXTURES}

In order to maintain the determinant different from zero the mass matrix for up quarks (or down quarks) has at most three texture-zeros. ${ }^{1}$ Also, we only have two types of realistic patterns depending on how the three texture zeros are distributed in the inputs of the mass matrix. In the first case we have a matrix with two texture zeros on the diagonal, and in the other case the matrix only contains a texture-zero on the diagonal, as it is pointed out in each column of Table I; where it is shown that by doing WB transformations with the permutation matrices $p_{i}$, with $i=1, \ldots, 6$, we obtain all possible viable cases for each pattern. Table I summarizes all the viable three-zero textures (via permutations) for the up and down quark mass matrices. Without loss of generality, as we will see later, we can write these patterns without including phases. An equivalence transformation through a permutation is a type of WB transformation, indeed, this fact allows us to find equivalent textures through permutations, for example

$$
\begin{aligned}
& M_{u}^{\prime}=\left(\begin{array}{ccc}
0 & \times & 0 \\
\times & \times & \times \\
0 & \times & \times
\end{array}\right)=p_{2} \cdot\left(\begin{array}{ccc}
0 & 0 & \times \\
0 & \times & \times \\
\times & \times & \times
\end{array}\right) \cdot p_{2}^{T}, \\
& M_{d}^{\prime}=\left(\begin{array}{lll}
0 & 0 & \times \\
0 & \times & \times \\
\times & \times & 0
\end{array}\right)=p_{2} \cdot\left(\begin{array}{ccc}
0 & \times & 0 \\
\times & 0 & \times \\
0 & \times & \times
\end{array}\right) \cdot p_{2}^{T},
\end{aligned}
$$

where " $x$ " stands for the nonzero entries. It is important to mention that the permutations do not change the number of zeros on the diagonal.

\footnotetext{
${ }^{1}$ More than three texture zeros implies that at least one quark mass is equal to zero or two of the quark masses must be equal [3,29].
} 
TABLE I. Mass matrix patterns with three texture-zeros. We are considering two cases, depending on the number of zeros in the diagonal (one or two texture zeros). It is not necessary to include phases.

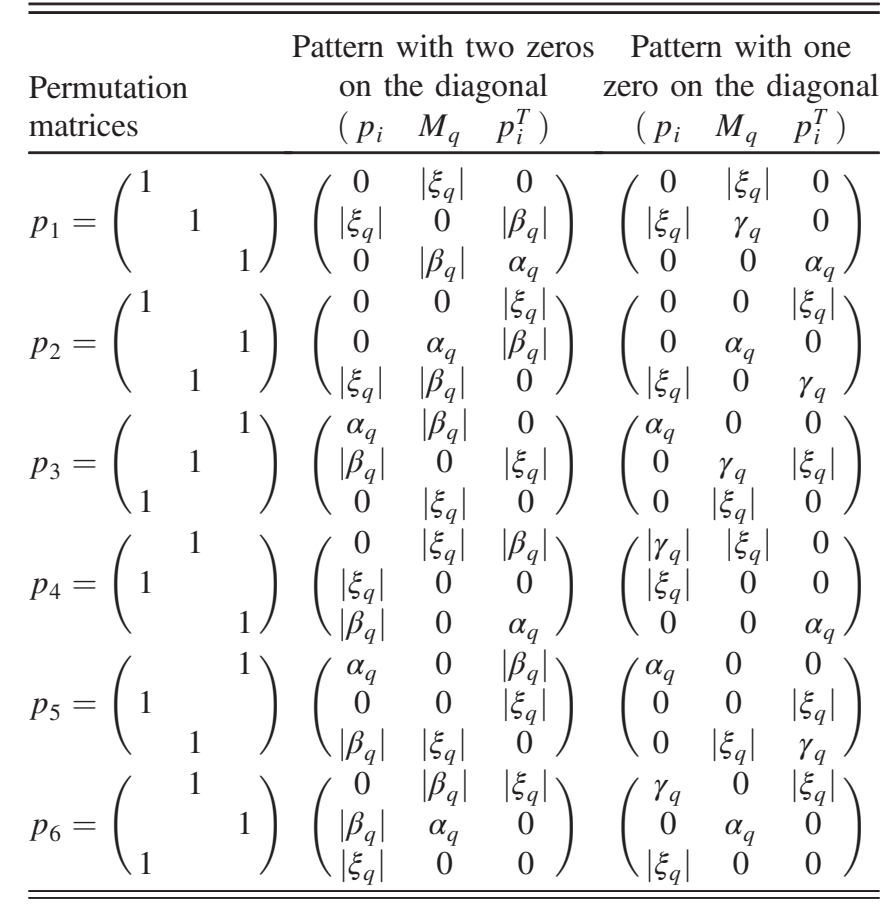

We will work with five-zero textures for the quark mass matrices. Six-zero textures have already been ruled out $[3,25,26,34]$.

\section{A. Texture-zero patterns}

The patterns shown in Table I can be analytically diagonalized. To accomplish this, we consider the most general case of a symmetric mass matrix with two texture zeros

$$
M_{q}=\left(\begin{array}{ccc}
0 & \left|\xi_{q}\right| & 0 \\
\left|\xi_{q}\right| & \gamma_{q} & \left|\beta_{q}\right| \\
0 & \left|\beta_{q}\right| & \alpha_{q}
\end{array}\right)
$$

where the phases of the off-diagonal parameters can be absorbed (or included) in only one of the mass matrices (the down-type or the up-type) through a WB transformation. $\gamma_{q}$ and $\alpha_{q}$ are real numbers due to the hermiticity of $M_{q}$. According to the Table I, the pattern with two zeros on the diagonal is achieved by making $\gamma_{q}=0$, and to obtain the pattern with a zero on the diagonal we set $\left|\beta_{q}\right|=0$. The mass matrix $M_{q}$ can be diagonalized using the transformation

$$
U_{q}^{\dagger} M_{q} U_{q}=D_{q}=\left(\begin{array}{lll}
\lambda_{1 q} & & \\
& \lambda_{2 q} & \\
& & \lambda_{3 q}
\end{array}\right),
$$

where the $\lambda_{i q}(i=1,2,3)$ are defined in (1.5). Note that $\gamma_{q}$, $\left|\beta_{q}\right|$ and $\left|\xi_{q}\right|$ can be expressed in terms of $\alpha_{q}$ and the $\lambda_{i q}$ 's. By using the invariants under a basis transformation, $\operatorname{tr} M_{q}$, $\operatorname{tr} M_{q}^{2}$ and $\operatorname{det} M_{q}$, it follows that

$$
\begin{aligned}
\gamma_{q} & =\lambda_{1 q}+\lambda_{2 q}+\lambda_{3 q}-\alpha_{q}, \\
\left|\beta_{q}\right| & =\sqrt{\frac{\left(\alpha_{q}-\lambda_{1 q}\right)\left(\alpha_{q}-\lambda_{2 q}\right)\left(\lambda_{3 q}-\alpha_{q}\right)}{\alpha_{q}}}, \\
\left|\xi_{q}\right| & =\sqrt{\frac{-\lambda_{1 q} \lambda_{2 q} \lambda_{3 q}}{\alpha_{q}}} .
\end{aligned}
$$

According to $[3,7,35]$ and the relation (2.4c) (which is real), $\alpha_{q}>0$; and from (2.4b), it must be found in one of the following intervals:

If $\lambda_{1 q}<0, \quad \lambda_{2 q}>0$ and $\lambda_{3 q}>0 \Rightarrow\left|\lambda_{2 q}\right| \leq \alpha_{q} \leq\left|\lambda_{3 q}\right|$

If $\lambda_{1 q}>0, \quad \lambda_{2 q}<0$ and $\lambda_{3 q}>0 \Rightarrow\left|\lambda_{1 q}\right| \leq \alpha_{q} \leq\left|\lambda_{3 q}\right|$.

If $\lambda_{1 q}>0, \quad \lambda_{2 q}>0$ and $\lambda_{3 q}<0 \Rightarrow\left|\lambda_{1 q}\right| \leq \alpha_{q} \leq\left|\lambda_{2 q}\right|$.

In the previous analysis, the (1.6) hierarchy was taken into account, and we only considered a negative eigenvalue according to the justification given in papers $[3,35] .^{2}$

The exact analytical matrix $U_{q}$, which diagonalizes the mass matrix $(2.2)$, is given by $[3,9,36]$

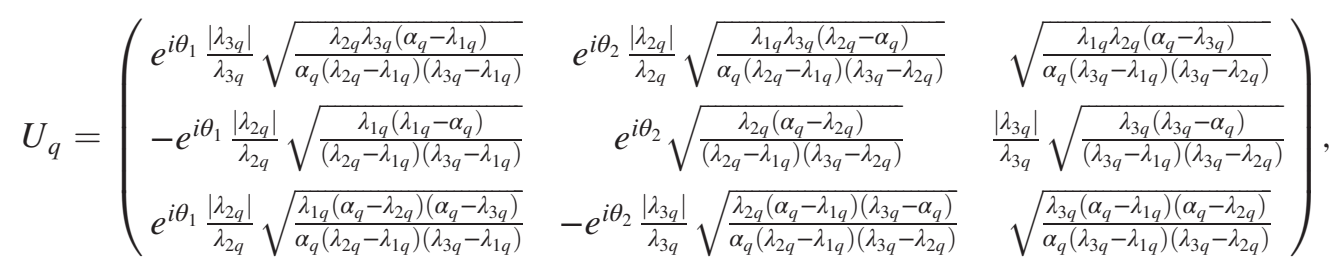

\footnotetext{
${ }^{2}$ The WB transformations allow us to use the basis (1.4a) (or the basis in (1.4b)) as the "starting point" matrices to generate any viable representation of quark mass matrices [3,11]. If there are texture zeros in mass matrices these can be found by a WB transformation. Texture zeros on the diagonal of the mass matrices imply that at least one of the proper values must be negative $[3,7,9,36]$.
} 
where we have included additional phases (nonphysical) to adjust the CKM mixing matrix to the usual convention (A2), as shown in the Ref. [12]. It is not necessary to include a phase in the third column, as it can be absorbed by the remaining phases.

The diagonalization matrix (2.6) can be seen as the unitary matrix of a WB transformation on the initial mass representations (1.4). For the case (1.4a):

$$
\begin{aligned}
& M_{u}^{\prime}=U_{u}\left(D_{u}\right) U_{u}^{\dagger}=\left(\begin{array}{ccc}
0 & \left|\xi_{u}\right| & 0 \\
\left|\xi_{u}\right| & \gamma_{u} & \left|\beta_{u}\right| \\
0 & \left|\beta_{u}\right| & \alpha_{u}
\end{array}\right), \\
& M_{d}^{\prime}=U_{u}\left(V D_{d} V^{\dagger}\right) U_{u}^{\dagger},
\end{aligned}
$$

where Eq. (2.3) was considered. As we have already mentioned, if we want a pattern of three zeros in the mass matrix $M_{u}^{\prime}$, with two zeros on the diagonal, that is, with $\gamma_{u}=0$, it is necessary to make $\alpha_{u}=\lambda_{1 u}+\lambda_{2 u}+\lambda_{3 u}$ according to (2.4a). From (2.5) this configuration is only possible for $\lambda_{1 u}, \lambda_{3 u}>0$, and $\lambda_{2 u}<0$. To find two additional texture zeros in the inputs of the mass matrix (2.7b), we adjust the free parameters $\theta_{1}$ and $\theta_{2}$ of the diagonalization matrix (2.6). On the other hand, if we want three zeros for the mass matrix
$M_{u}^{\prime}$, but with a single zero on the diagonal, it is necessary to set $\left|\beta_{u}\right|=0$. To achieve this we have three possibilities [from Eq. (2.4b)]: $\alpha_{u}=\lambda_{1 u}$, or $\alpha_{u}=\lambda_{2 u}$, or $\alpha_{u}=\lambda_{3 u}$. In each of these cases, one of the remaining $\lambda_{i u}$ 's must be negative, which gives a total of six different possibilities. A similar exercise can be carried out in the case (1.4b).

$$
\begin{gathered}
M_{u}^{\prime}=U_{d}\left(V^{\dagger} D_{u} V\right) U_{d}^{\dagger}, \\
M_{d}^{\prime}=U_{d}\left(D_{d}\right) U_{d}^{\dagger}=\left(\begin{array}{ccc}
0 & \left|\xi_{d}\right| & 0 \\
\left|\xi_{d}\right| & \gamma_{d} & \left|\beta_{d}\right| \\
0 & \left|\beta_{d}\right| & \alpha_{d}
\end{array}\right) .
\end{gathered}
$$

where we have used the relation (2.3) for the special case $q=d$. Table II summarizes the numerical results of our study, in the next section we will see these results in more detail from an analytical point of view.

\section{MASS MATRICES WITH FIVE TEXTURE ZEROS}

As it is well known in the literature, for a given texture it is possible to establish relations between the quark masses, the mixing angles and the $C P$ violation phase of the CKM matrix, so that, a study of these relations is important to shed light on

TABLE II. Patterns for quark mass matrices with five texture zeros. The Wolfenstein parameters for the CKM mixing matrix and the quark masses are reproduced with deviations below $1 \sigma$ level. In the last column $P_{A}=\frac{A_{\mathrm{WB}}-A_{\mathrm{PDG}}}{\Delta A}$, where $A_{\mathrm{WB}}$ and $A_{\mathrm{PDG}}$ are the values for $A$ from the WB transformation and the PDG best fit, respectively. $\Delta A$ is the uncertainty for $A$ reported in the PDG.

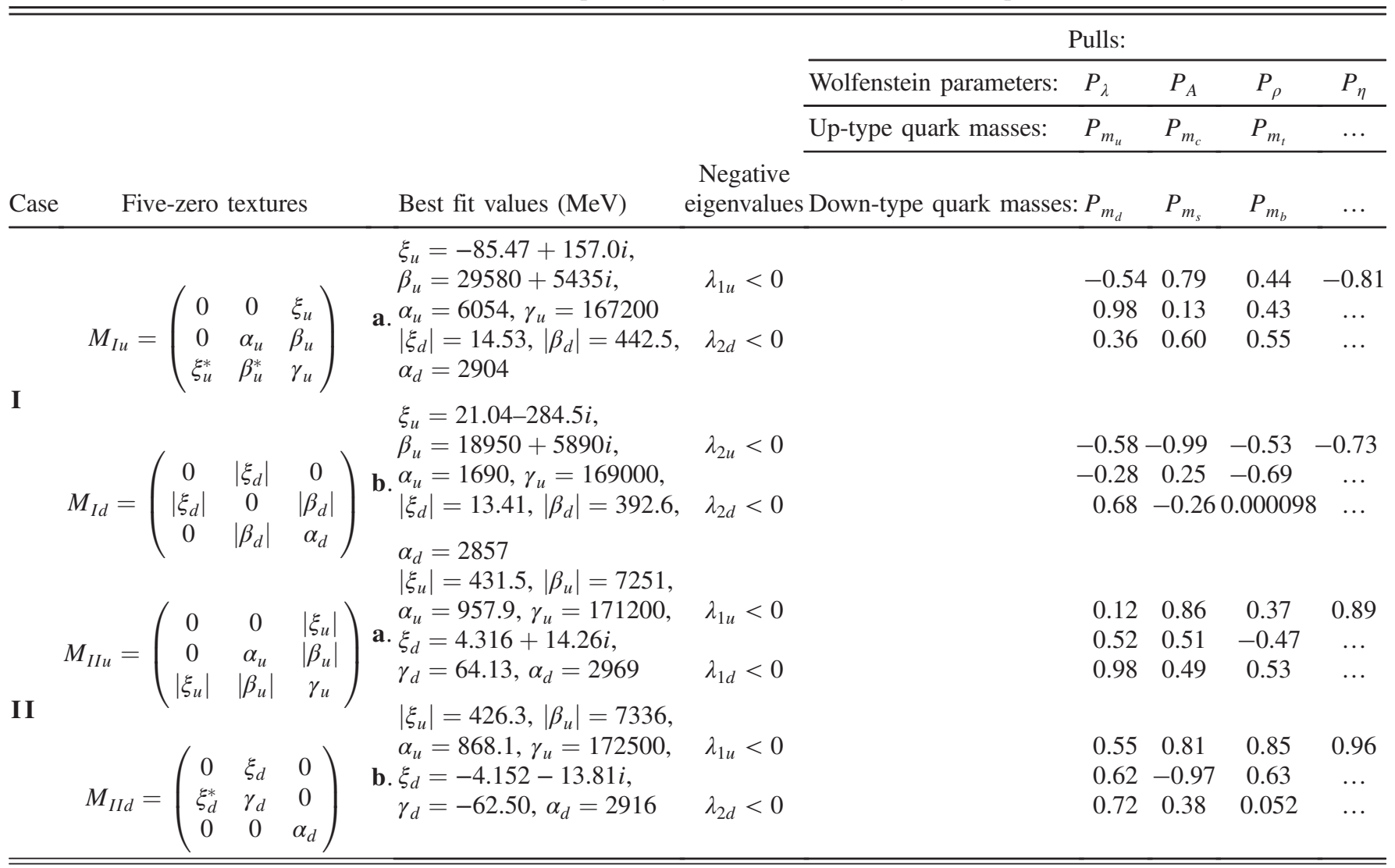


the underlying symmetries of the flavor physics. The five-zero textures for the quark mass matrices given in Table II are viable models according to the latest data for the current quark masses and the CKM mixing matrix parameters at the $Z$ scale. In what follows we will consider various cases to implement quark mass matrices with five texture-zeros.

\section{A. Case I}

In this configuration, the down-type quark mass matrix contains three texture zeros, two of them on the diagonal, corresponding to the case I in Table II, which has the following analytical structure for quark mass matrices

$$
\begin{aligned}
M_{I u} & =P^{\dagger}\left(\begin{array}{ccc}
0 & 0 & \left|\xi_{u}\right| \\
0 & \alpha_{u} & \left|\beta_{u}\right| \\
\left|\xi_{u}\right| & \left|\beta_{u}\right| & \gamma_{u}
\end{array}\right) P, \\
M_{I d} & =\left(\begin{array}{ccc}
0 & \left|\xi_{d}\right| & 0 \\
\left|\xi_{d}\right| & 0 & \left|\beta_{d}\right| \\
0 & \left|\beta_{d}\right| & \alpha_{d}
\end{array}\right)
\end{aligned}
$$

where all the phases are reduced to those contained in the diagonal matrix $P=\operatorname{diag}\left(e^{-i \phi_{\xi_{u}}}, e^{-i \phi_{\beta_{u}}}, 1\right)$ [with $\phi_{\beta_{u}} \equiv$ $\arg \left(\beta_{u}\right)$ and $\left.\phi_{\xi_{u}} \equiv \arg \left(\xi_{u}\right)\right]$ which comes from doing a WB transformation, in such a way that the phases of $M_{I d}$ are absorbed in $P$. So we have 7 real parameters and 2 phases, to reproduce 10 physical quantities: 6 quark masses, 3 mixing angles and the $C P$ violating phase of the CKM mixing matrix, which implies that relations between masses and mixing angles can be established in the quark sector. The five-zero texture deduced in (3.1) is not a Fritzsch texture of those studied in [1]. Even though they are not identical, the mass matrices (3.1) can be diagonalized with the help of the matrix (2.6). Let us use the permutation matrix $P_{2}=[(1,0,0),(0,0,1),(0,1,0)]$, to bring the up-type quark mass matrix to the form
$M_{u}=P^{\dagger} P_{2}\left(\begin{array}{ccc}0 & \left|\xi_{u}\right| & 0 \\ \left|\xi_{u}\right| & \gamma_{u} & \left|\beta_{u}\right| \\ 0 & \left|\beta_{u}\right| & \alpha_{u}\end{array}\right) P_{2} P$, in such a way that the internal matrix corresponds to that in (2.2). Therefore, the diagonalization matrix is the unitary matrix $P^{\dagger} P_{2} U_{u}$, where $U_{u}$ is defined in (2.6), for the case $q=u$. According to $(2.4 \mathrm{a})$ the other mass matrix in (3.1), $M_{I d}$, can be diagonalized if we make $\alpha_{d}=\lambda_{1 d}+\lambda_{2 d}+\lambda_{3 d}$.

From (2.4) the mass matrix parameters are

$$
\begin{aligned}
\gamma_{u} & =\mp m_{u} \pm m_{c}+m_{t}-\alpha_{u}, \\
\left|\beta_{u}\right| & =\sqrt{\frac{\left(\alpha_{u} \pm m_{u}\right)\left(\alpha_{u} \mp m_{c}\right)\left(m_{t}-\alpha_{u}\right)}{\alpha_{u}},} \\
\left|\xi_{u}\right| & =\sqrt{\frac{m_{u} m_{c} m_{t}}{\alpha_{u}}}, \\
\alpha_{d} & =m_{d}-m_{s}+m_{b}, \\
\left|\beta_{d}\right| & =\sqrt{\frac{\left(m_{b}-m_{s}\right)\left(m_{d}+m_{b}\right)\left(m_{s}-m_{d}\right)}{m_{d}-m_{s}+m_{b}}}, \\
\left|\xi_{d}\right| & =\sqrt{\frac{m_{d} m_{s} m_{b}}{m_{d}-m_{s}+m_{b}}},
\end{aligned}
$$

where for the eigenvalues of $M_{I u}$ we have considered two possible cases $\lambda_{1 u}<0$ (upper sign) and $\lambda_{2 u}<0$ (lower sign). $\alpha_{u}$ is a free parameter which, according to the Eqs. (2.5), takes values in the intervals:

$$
\begin{aligned}
& m_{c} \leq \alpha_{u} \leq m_{t} \quad \text { for } \lambda_{1 u}<0, \\
& m_{u} \leq \alpha_{u} \leq m_{t} \quad \text { for } \lambda_{2 u}<0 .
\end{aligned}
$$

The diagonalization matrices for $M_{I u}$ and $M_{I d}$ in (3.1) are

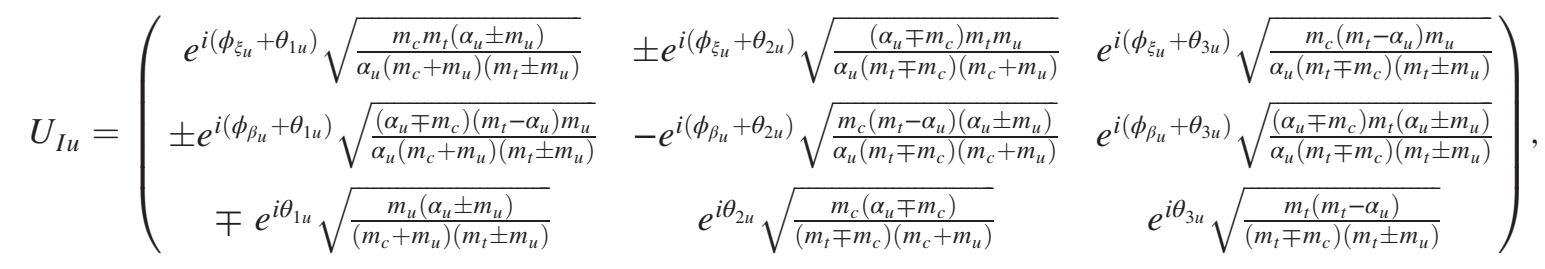

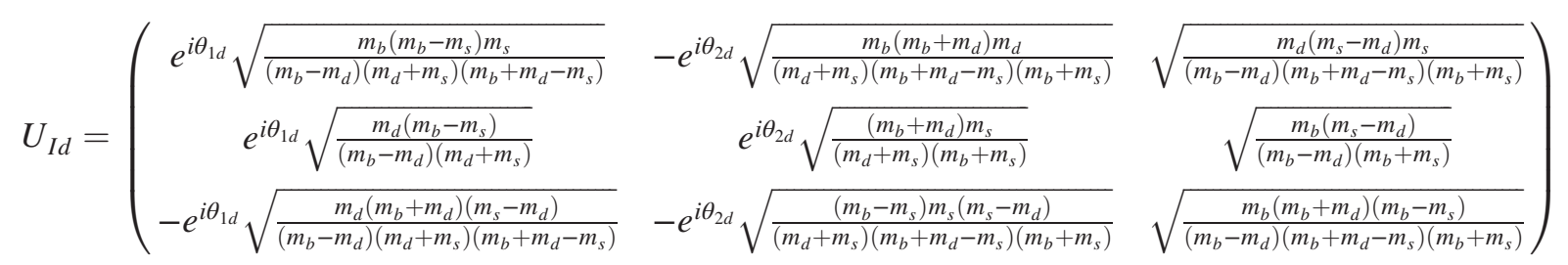


where the nonphysical phases $\theta_{1 u}, \theta_{2 u}, \theta_{3 u}, \theta_{1 d}$ and $\theta_{2 d}$ are necessary in order to adjust our theoretical prediction for the CKM to the established convention. To obtain the leading order (LO) terms that contribute to the CKM mixing matrix $V=U_{I u}^{\dagger} U_{I d}$ we use the hierarchy of the quark masses (1.6). The analytical results for the LO CKM entries are summarized in Table $\mathrm{V}$. There are several aspects to highlight about the case I:

(i) In the SM the inputs $\left|V_{c s}\right| \approx\left|V_{t b}\right| \approx 1$ then the free parameter must satisfy $\alpha_{u} \ll m_{t}$, hence $\alpha_{u} / m_{t} \ll 1$. Also, due to the condition (3.3a), we have $\alpha_{u} \gg m_{u}$.

(ii) The free parameter $\alpha_{u} / m_{t}$ is only relevant for the real parts of the matrix elements $V_{t b}$ (although $\alpha_{u} / m_{t} \ll 1$ this matrix element is very precisely determined) and $V_{u b}$. For the matrix elements $V_{t s}$, $V_{c b}, V_{u b}$ and $V_{t d}, \alpha_{u} / m_{t}$ is relevang for adjusting the $C P$ violating phase. For the remaining matrix elements, by neglecting linear terms in $\alpha_{u} / m_{t}$ and $m_{c} / m_{t}$, the dominant contributions only depend on ratios between down-type quark masses.

(iii) Relations can be established between the elements of the mixing matrix whose LO terms only involve quark masses as shown in Table IV. Some of these relations are well known, for example the GattoSartori-Tonin (GST) (Eq. (2), in Table IV) [37]: $\tan \theta_{12}=\left|V_{u s} / V_{u d}\right|=\sqrt{m_{d} / m_{s}}$, which is approximately fulfilled. Another important relation that we can find and that is successfully verified, according to the experimental result (1.7), is given by the expression: $\left|V_{t d} / V_{t s}\right| \approx \sqrt{m_{d} / m_{s}}$ [38]. On the other hand, our analysis also allows us to establish that the relation $\left|V_{u b} / V_{c b}\right|$ does not coincide with the result $\sqrt{m_{u} / m_{c}}$, in accordance with the experimental data (1.7).

(iv) The best fits for the mass matrix parameters (3.1) are shown in Table III.

\section{B. Case II}

Another viable analytical texture in Table II is the case II, with quark mass matrices given by

$$
\begin{aligned}
M_{I I u} & =\left(\begin{array}{ccc}
0 & 0 & \left|\xi_{u}\right| \\
0 & \alpha_{u} & \left|\beta_{u}\right| \\
\left|\xi_{u}\right| & \left|\beta_{u}\right| & \gamma_{u}
\end{array}\right), \\
M_{I I d} & =\left(\begin{array}{ccc}
0 & \left|\xi_{d}\right| e^{i \phi_{\xi_{d}}} & 0 \\
\left|\xi_{d}\right| e^{-i \phi_{\xi_{d}}} & \gamma_{d} & 0 \\
0 & 0 & \alpha_{d}
\end{array}\right) .
\end{aligned}
$$

In this case we have only one phase, $\phi_{\xi_{d}}$, responsible for the $C P$ violation. And there are 7 real parameters. This texture is a Fritzsch-type [1].
TABLE III. Fit parameters.

\begin{tabular}{lccccc}
\hline \hline & \multicolumn{2}{c}{ Case I } & & \multicolumn{2}{c}{ Case II } \\
\cline { 2 - 3 } \cline { 5 - 6 } & $\lambda_{1 u}<0$ & $\lambda_{2 u}<0$ & & $\lambda_{1 u}<0$ & $\lambda_{1 u}<0$ \\
& $\lambda_{2 d}<0$ & $\lambda_{2 d}<0$ & & $\lambda_{1 d}<0$ & $\lambda_{2 d}<0$ \\
\hline$\theta_{1 u}$ & -1.423 & -2.844 & -1.975 & -1.991 \\
$\theta_{2 u}$ & 0.6701 & 1.856 & 0 & 0 \\
$\theta_{3 u}$ & -0.004737 & -0.004617 & 0 & 0 \\
$\theta_{1 d}$ & 0.6360 & 1.930 & 3.025 & -0.1351 \\
$\theta_{2 d}$ & -2.285 & -0.9766 & 3.148 & 3.148 \\
$\phi_{\xi_{u}}$ & 2.069 & -1.497 & & $\ldots$ & $\ldots$ \\
$\phi_{\beta_{u}}$ & 0.1817 & 0.3015 & & $\ldots$ & $\ldots$ \\
$\phi_{\xi_{d}}$ & $\ldots$ & $\ldots$ & & 1.277 & -1.863 \\
$\alpha_{u}(\mathrm{MeV})$ & 6054 & 1690 & 957.9 & 868.1 \\
$m_{u}(\mathrm{MeV})$ & 1.792 & 1.268 & & 1.599 & 1.642 \\
$m_{c}(\mathrm{MeV})$ & 625.5 & 633.2 & 650.2 & 555.7 \\
$m_{t}(\mathrm{MeV})$ & 172600 & 171300 & 171500 & 172900 \\
$m_{d}(\mathrm{MeV})$ & 2.993 & 3.148 & 3.292 & 3.166 \\
$m_{s}(\mathrm{MeV})$ & 68.93 & 56.12 & 67.42 & 65.66 \\
$m_{b}(\mathrm{MeV})$ & 2970 & 2910 & 2969 & 2916 \\
\hline \hline
\end{tabular}

TABLE IV. Leading order relations between the CKM matrix elements.

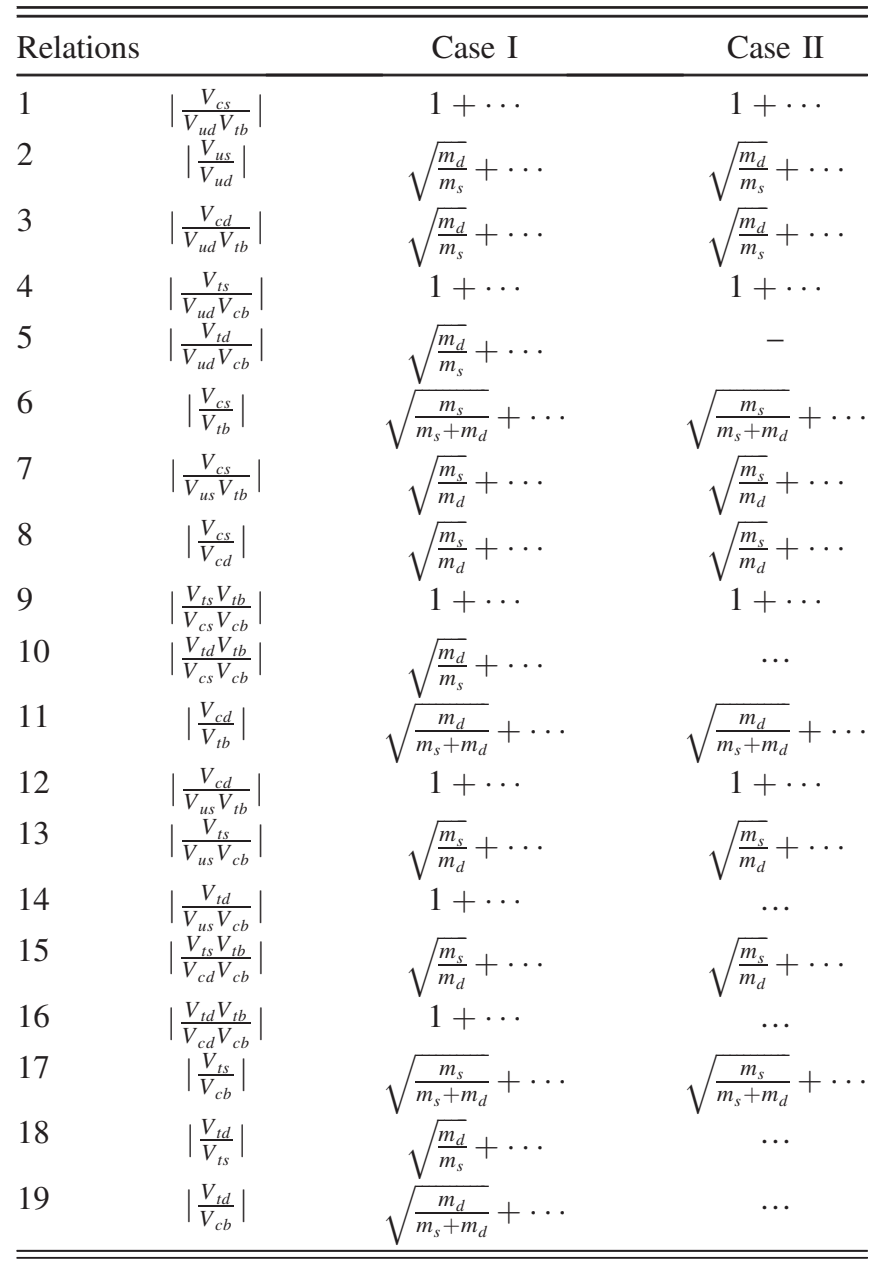


As in the previous case we can obtain relations between the elements of the CKM and the quark masses. The structure of the matrix $M_{I I u}$ is similar to the one given in Eq. (3.1), without including phases, so it can be inferred that the diagonalization matrix for this case is: $P_{2} U_{u}$ with $P_{2}=[(1,0,0),(0,0,1),(0,1,0)]$ and $U_{u}$ defined in (2.6) for $q=u$.

The matrix $M_{I I d}$ in (3.6) has a zero structure like the one given in (2.2) with $\left|\beta_{q}\right|=0$, so that, there are several possibilities to be considered: $\alpha_{d}=\lambda_{1 d}>0$, $\left|\xi_{d}\right|=\sqrt{-\lambda_{2 d} \lambda_{3 d}}$ and $\gamma_{d}=\lambda_{2 d}+\lambda_{3 d} ;$ or $\alpha_{d}=\lambda_{2 d}>0$, $\left|\xi_{d}\right|=\sqrt{-\lambda_{1 d} \lambda_{3 d}}$ and $\gamma_{d}=\lambda_{1 d}+\lambda_{3 d}$; or $\alpha_{d}=\lambda_{3 d}>0$, $\left|\xi_{d}\right|=\sqrt{-\lambda_{1 d} \lambda_{2 d}}$ and $\gamma_{d}=\lambda_{1 d}+\lambda_{2 d}$ [3]. From the last option we obtain the two cases with the best agreement with the data, as reported in Table II. Here the diagonalization matrix for $M_{I I d}$ is $P_{d}^{\dagger} U_{d}$ with $U_{d}$ as given in (2.6) for $q=d$ and $P_{d}=\operatorname{diag}\left(e^{-i \phi_{\xi_{d}}}, 1,1\right)$. The parameters of the mass matrices (3.6), according to the relations (2.4) are given by:

$$
\gamma_{u}=-m_{u}+m_{c}+m_{t}-\alpha_{u},
$$

$$
\begin{gathered}
\left|\beta_{u}\right|=\sqrt{\frac{\left(\alpha_{u}+m_{u}\right)\left(\alpha_{u}-m_{c}\right)\left(m_{t}-\alpha_{u}\right)}{\alpha_{u}},} \\
\left|\xi_{u}\right|=\sqrt{\frac{m_{u} m_{c} m_{t}}{\alpha_{u}}}, \\
\alpha_{d}=m_{b}, \\
\left|\xi_{d}\right|=\sqrt{m_{d} m_{s}}, \\
\gamma_{d}=\mp m_{d} \pm m_{s},
\end{gathered}
$$

where $\lambda_{1 u}<0$; the upper sign, for $\lambda_{1 d}<0$ and the lower sign, for $\lambda_{2 d}<0$; and $\alpha_{u}>0$ is a free parameter in the range:

$$
m_{c} \leq \alpha_{u} \leq m_{t}
$$

In this case, the diagonalization matrices of the mass operators (3.6), are

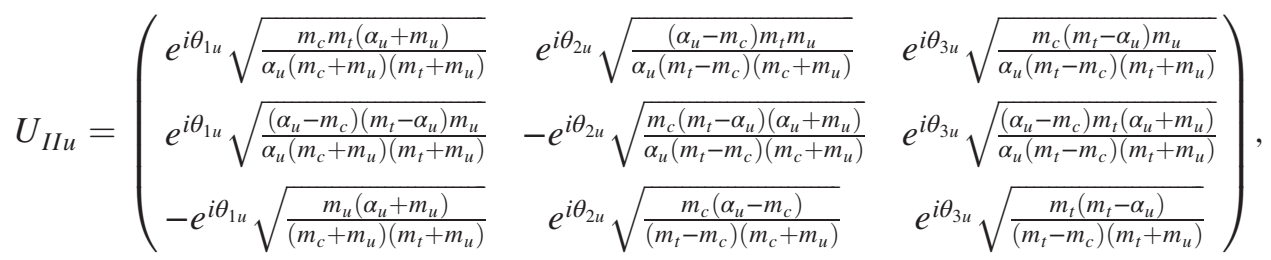

$U_{I I d}=\left(\begin{array}{ccc}e^{i\left(\phi_{\xi_{d}}+\theta_{1 d}\right)} \sqrt{\frac{m_{s}}{m_{d}+m_{s}}} & \pm e^{i\left(\phi_{\xi_{d}}+\theta_{2 d}\right)} \sqrt{\frac{m_{d}}{m_{d}+m_{s}}} & 0 \\ \mp e^{i \theta_{1 d}} \sqrt{\frac{m_{d}}{m_{d}+m_{s}}} & e^{i \theta_{2 d} \sqrt{\frac{m_{s}}{m_{d}+m_{s}}}} & 0 \\ 0 & 0 & 1\end{array}\right)$.

The best fit parameters are shown in Table III.

Taking into account the hierarchy of the quark masses, Eq. (1.6), and the interval for the parameter $\alpha_{u}$, Eq. (3.8), to LO the entries of the CKM, $V=U_{u}^{\dagger} U_{d}$, are summarized in Table V, case II; from these results we conclude that:

(i) As in the case I, $\left|V_{c s}\right| \approx\left|V_{t b}\right| \approx 1$, such that $\alpha_{u} \ll m_{t}$. Also, due to (3.8), $\alpha_{u} \gg m_{u}$. Again we verify that the relation $\left|V_{u b} / V_{c b}\right| \neq \sqrt{m_{u} / m_{c}}$ is not satisfied. Although the relationship $\left|V_{t d} / V_{t s}\right| \approx$ $\sqrt{m_{d} / m_{s}}$ is not verified in principle for this case; we can see from Table V, for the case II, that if we omit the second term of the approximation for $V_{t d}$, it is enough to reproduce this relationship. As it is shown numerically, for this case high order contributions are negligible. (ii) The CKM matrix elements, $V_{t s}, V_{c b}, V_{u b}, V_{t d}$ depend heavily on the $\alpha_{u}$ parameter, the remaining elements depend on ratios between down quark masses. Only the $V_{t d}$ matrix element has information about the phase, which in turn depends on the ratio $\alpha_{u} / m_{t}$, which is a noticeable difference with respect to case I.

(iii) As in the case I, the LO relations between the CKM elements involving only quark masses are shown in Table IV.

(iv) Although, the results are similar to the expressions given in Table IV for the case I, for the case II the relations 5, 10, 14, 16, 18 and 19 are absent (the corresponding expressions are cumbersome).

\section{CONCLUSIONS}

Using the WB transformation method [3,11], we found configurations for the quark mass matrices with the maximum number of possible texture zeros. To accomplish this, we start from the general basis (1.4a) and (1.4b), from which the expressions (2.7) and (2.8) can be obtained, respectively. Modulo permutations, only the configurations shown in Table I, for mass matrices with one or two zeros in 
the diagonal, are possible. From these patterns we obtained the cases I and II in Table II, corresponding to the five-zero textures in Eq. (3.1) and Eq. (3.6), respectively, which reproduce the quark masses, mixing angles and the $C P$ violation phase, with deviations from the experimental values below $1 \sigma$ level.

The first case has nine free parameters: 7 real and 2 phases, while the second case has eight free parameters: 7 real and 1 phase. In both cases, it is necessary to reproduce ten physical quantities: 6 quark masses, 3 mixing angles and the $C P$ violation phase, the lack of balance between the number of free parameters and the physical quantities implies physical relations between the quark masses and the CKM mixing angles, which are reported in Table V. Additionally, the relation GST [37] is maintained and we can adjust the $C P$ violation phase of the SM. Additionally, our five-zero texture models reproduce the experimental quantities (1.7) deviating from the experimental central value by at most $1 \sigma$ level. First, for both cases I and II, we can verify that the relation $\left|V_{t d} / V_{t s}\right| \approx \sqrt{m_{d} / m_{s}}$ is satisfied, while the relation $\left|V_{u b} / V_{c b}\right| \neq \sqrt{m_{u} / m_{c}}$ is not, as already indicated in previous works [20-22]. In some cases, even with the LO approximations given in Table $\mathrm{V}$ we can reproduce the results (1.7). We have several free parameters to adjust the physical quantities: the $C P$-violating phases, the calibration phases $\theta_{q i}$ with $q=u, d$, in the diagonalization matrices, and the real parameter $\alpha_{u}$. In our analysis the analytical LO expressions for the case I, with $\lambda_{1 u}<0$ and for the case II, with $\lambda_{1 d}<0$, are enough to keep all the observables inside of the error bars. For the other cases, $\lambda_{2 u}<0$ and $\lambda_{2 d}<0$, satisfactory results were not achieved with the LO approximations provided in Table V. In these cases, the complete expressions must be taken into account in order to get a good fit.

The case I is an original proposal which was not considered in the Fritzsch original work [1] nor in later studies. Case II has been widely considered in the literature $[1,5,23,25,30,31]$, but in our approach, we take a negative eigenvalue (which has not been considered previously) for the mass of the lightest down quark, that is, $\lambda_{1 d}<0$. Here, it should be mentioned that, without losing generality, only one negative eigenvalue is necessary for each mass matrix $[3,11]$. Also, it is important to say that the relations in Table $\mathrm{V}$ are comparable to the results reported in [30,31,34,36,39].

The purpose of the texture zeros for quark mass matrices is to find relations between quark masses and the flavor mixing parameters in consistency with the experimental data [1]. For the textures deduced in this work, the quark mass ratios contribute significantly to the flavor mixing parameters as shown in Table IV; In Table V, it is possible to observe additional contributions (not exclusively dependent on the quark masses) which also depend on the free parameter $\alpha_{\mu}$ and on the phases responsible for the $C P$ violation. It is important to highlight that the LO contributions to the relations involving the CKM matrix elements mainly depend on ratios of down-type quark masses. The relations reported in this manuscript, could be useful to disentangle the underlying symmetries under the mass scales in the SM.

\section{ACKNOWLEDGMENTS}

In these subjects we acknowledge the collaboration of W. A. Ponce, R. Benavides and L. Muñoz. We also acknowledge financial support from VIIS in the Universidad de Nariño, Approval Contract No. 024 and 160, project numbers: 1048, 1928 and 2172.

\section{APPENDIX: QUARK MASS MATRICES AND THE CKM MIXING MATRIX}

The parameters of the CKM are reported at the $Z$ pole scale $\mu=M_{Z}$, hence the same scale is used to evaluate the current quark masses (in $\mathrm{MeV}$ ) [19]., i.e.,

$$
\begin{aligned}
& m_{u}=1.38_{-0.41}^{+0.42}, \quad m_{c}=638_{-84}^{+43}, \quad m_{t}=172100 \pm 1200, \\
& m_{d}=2.82 \pm 0.48, \quad m_{s}=57_{-12}^{+18}, \quad m_{b}=2860_{-60}^{+160} .
\end{aligned}
$$

The CKM unitary matrix $[22,40,41]$ can be parameterized by three mixing angles and the $C P$ violation phase [41]. The form of this matrix in the standard parametrization is given by [42].

$$
V=\left(\begin{array}{ccc}
V_{u d} & V_{u s} & V_{u b} \\
V_{c d} & V_{c s} & V_{c b} \\
V_{t d} & V_{t s} & V_{t b}
\end{array}\right)=\left(\begin{array}{ccc}
c_{12} c_{13} & s_{12} c_{13} & s_{13} e^{-i \delta} \\
-s_{12} c_{23}-c_{12} s_{23} s_{13} e^{i \delta} & c_{12} c_{23}-s_{12} s_{23} s_{13} e^{i \delta} & s_{23} c_{13} \\
s_{12} s_{23}-c_{12} c_{23} s_{13} e^{i \delta} & -c_{12} s_{23}-s_{12} c_{23} s_{13} e^{i \delta} & c_{23} c_{13}
\end{array}\right),
$$

where $s_{i j}=\sin \theta_{i j}, c_{i j}=\cos \theta_{i j}$, the angles $\theta_{i j}$ are said to lie in the first quadrant, such that $\sin \theta_{i j}, \cos \theta_{i j} \geq 0$. The phase $\delta$ is responsible for all the $C P$ violation phenomena in the flavor changing processes in SM. For various applications it is useful to use the Wolfenstein parametrization [22]

$$
\begin{aligned}
& \lambda=\sin \theta_{12}, \quad A=\frac{\sin \theta_{23}}{\sin ^{2} \theta_{12}}, \quad \rho=\frac{\sin \theta_{13} \cos \delta}{\sin \theta_{12} \sin \theta_{23}}, \\
& \eta=\frac{\sin \theta_{13} \sin \delta}{\sin \theta_{12} \sin \theta_{23}} .
\end{aligned}
$$


TABLE V. Cases I and II for the quark mass matrices with five texture-zeros. And their corresponding LO predictions for the CKM elements.

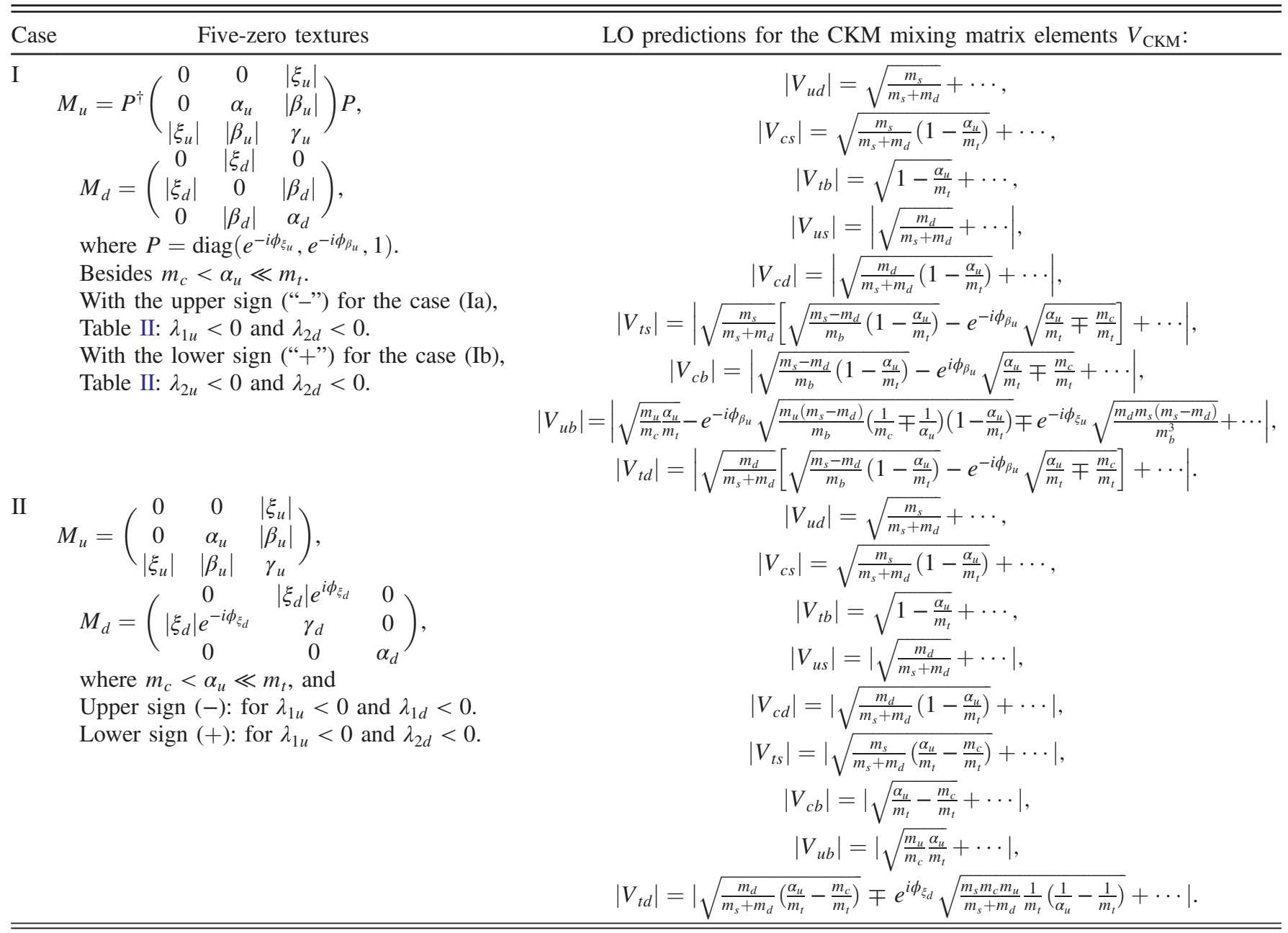

The CKMfitter and UTfit Collaborations [20,21] provide updated fits for the Wolfenstein parameters,

$$
\lambda=0.22500_{-0.00100}^{+0.00100}, \quad A=0.826_{-0.012}^{+0.012}, \quad \rho=0.152_{-0.014}^{+0.014}, \quad \eta=0.357_{-0.010}^{+0.010} .
$$

The best fit values for CKM matrix elements are

$$
V=\left(\begin{array}{ccc}
(0.97431 \pm 0.00012) & (0.22514 \pm 0.00055) & (0.00365 \pm 0.00010) e^{i(-66.8 \pm 2.0)^{\circ}} \\
(-0.22500 \pm 0.00054) e^{i(0.0351 \pm 0.0010)^{\circ}} & (0.97344 \pm 0.00012) e^{i(-0.001880 \pm 0.000052)^{\circ}} & (0.04241 \pm 0.00065) \\
(0.00869 \pm 0.00014) e^{i(-22.23 \pm 0.63)^{\circ}} & (-0.04124 \pm 0.00056) e^{i(1.056 \pm 0.032)^{\circ}} & (0.999112 \pm 0.000024)
\end{array}\right)
$$


[1] H. Fritzsch and Z.-Z. Xing, Mass and flavor mixing schemes of quarks and leptons, Prog. Part. Nucl. Phys. 45, 1 (2000).

[2] H. Fusaoka and Y. Koide, Updated estimate of running quark masses, Phys. Rev. D 57, 3986 (1998).

[3] Y. Giraldo, Texture zeros and WB transformations in the quark sector of the Standard Model, Phys. Rev. D 86, 093021 (2012).

[4] M. Gupta and G. Ahuja, Flavor mixings and textures of the fermion mass matrices, Int. J. Mod. Phys. A 27, 1230033 (2012)

[5] P. O. Ludl and W. Grimus, A complete survey of texture zeros in general and symmetric quark mass matrices, Phys. Lett. B 744, 38 (2015).

[6] Z.-Z. Xing and Z.-H. Zhao, On the four-zero texture of quark mass matrices and its stability, Nucl. Phys. B897, 302 (2015).

[7] G. C. Branco, D. Emmanuel-Costa, and R. G. Felipe, Texture zeros and weak basis transformations, Phys. Lett. B 477, 147 (2000).

[8] R. Verma, Minimal weak basis textures and quark mixing data, J. Phys. G 40, 125003 (2013).

[9] Z.Z. Xing and H. Zhang, Complete parameter space of quark mass matrices with four texture zeros, J. Phys. G 30, 129 (2004).

[10] Y.-F. Zhou, Textures and hierarchies in quark mass matrices with four texture zeros, arXiv:hep-ph/0309076.

[11] Y. Giraldo, Seeking texture zeros in the quark mass matrix sector of the standard model, Nucl. Part. Phys. Proc. 267-269, 76 (2015).

[12] Y. Giraldo, Reply to "Comment on "Texture Zeros and WB transformations in the quark sector of the Standard Model". Phys. Rev. D 91, 038302 (2015).

[13] H. Fritzsch, Calculating the cabibbo angle, Phys. Lett. 70B, 436 (1977).

[14] H. Fritzsch, Quark masses and flavor mixing, Nucl. Phys. B155, 189 (1979).

[15] S. Weinberg, The problem of mass, Trans. N.Y. Acad. Sci. 38, 185 (1977).

[16] H. Fritzsch, Weak interaction mixing in the six-quark theory, Phys. Lett. 73B, 317 (1978).

[17] H. Fritzsch and Z.-Z. Xing, Four zero texture of Hermitian quark mass matrices and current experimental tests, Phys. Lett. B 555, 63 (2003).

[18] B. Dutta and S. Nandi, A new ansatz: Fritzsch mass matrices with least modification, Phys. Lett. B 366, 281 (1996).

[19] Z.-Z. Xing, H. Zhang, and S. Zhou, Impacts of the Higgs mass on vacuum stability, running fermion masses and twobody Higgs decays, Phys. Rev. D 86, 013013 (2012).

[20] U. Collaboration, Utfit, http://www.utfit.org/UTfit/ (2019).

[21] T. C. Group, Ckmfitter, http://ckmfitter.in2p3.fr/www/html/ ckm_main.html (2019).

[22] M. Tanabashi et al., Review of particle physics, Phys. Rev. D 98, 030001 (2018).
[23] B. R. Desai and A. R. Vaucher, Quark mass matrices with four and five texture zeroes, and the CKM matrix, in terms of mass eigenvalues, Phys. Rev. D 63, 113001 (2001).

[24] P. Fakay, Revisiting texture 5 zero quark mass matrices, arXiv:1410.7142.

[25] N. Mahajan, R. Verma, and M. Gupta, Investigating nonFritzsch like texture specific quark mass matrices, Int. J. Mod. Phys. A 25, 2037 (2010).

[26] M. Randhawa, V. Bhatnagar, P. S. Gill, and M. Gupta, Unique mass texture for quarks and leptons, Phys. Rev. D 60, 051301 (1999).

[27] S. Sharma, P. Fakay, G. Ahuja, and M. Gupta, Clues towards unified textures, Int. J. Mod. Phys. A 29, 1444005 (2014).

[28] S. Sharma, P. Fakay, G. Ahuja, and M. Gupta, Finding a unique texture for quark mass matrices, Phys. Rev. D 91, 053004 (2015).

[29] W. A. Ponce and R. H. Benavides, Texture zeros for the standard model quark mass matrices, Eur. Phys. J. C 71, 1641 (2011).

[30] W. A. Ponce, J. D. Gómez, and R. H. Benavides, Five texture zeros and $C P$ violation for the standard model quark mass matrices, Phys. Rev. D 87, 053016 (2013).

[31] R. Verma, Exploring the predictability of symmetric texture zeros in quark mass matrices, Phys. Rev. D 96, 093010 (2017).

[32] H. Fritzsch, Hierarchical chiral symmetries and the quark mass matrix, Phys. Lett. B 184, 391 (1987).

[33] A. E. C. Hernández and I. de Medeiros Varzielas, Viable textures for the fermion sector, J. Phys. G 42, 065002 (2015).

[34] S. Kaundal, A. Bagai, G. Ahuja, and M. Gupta, Minimal texture of quark mass matrices and precision CKM measurements (2019).

[35] Y. Giraldo and E. Rojas, Five non-fritzsch texture zeros for quarks mass matrices in the standard model, in 38th International Symposium on Physics in Collision (2018).

[36] H. Fritzsch and Z.. Z. Xing, Four zero texture of hermitian quark mass matrices and current experimental tests, Phys. Lett. B 555, 63 (2003).

[37] R. Gatto, G. Sartori, and M. Tonin, Weak self-masses, Cabibbo angle, and broken $s u(2) \otimes s u(2)$, Phys. Lett. 28B, 128 (1968).

[38] H. Fritzsch, Flavor mixing of quarks and a new texture, Mod. Phys. Lett. A 36, 2150097 (2021).

[39] P. S. Gill and M. Gupta, Fritzsch-xing mass matrices, $v_{t d}$ and $C P$ violating phase $\delta$, Phys. Rev. D 56, 3143 (1997).

[40] N. Cabibbo, Unitary Symmetry and Leptonic Decays, Phys. Rev. Lett. 10, 531 (1963).

[41] M. Kobayashi and T. Maskawa, Cp violation in the renormalizable theory of weak interaction, Prog. Theor. Phys. 49, 652 (1973).

[42] L.-L. Chau and W.-Y. Keung, Comments on the Parametrization of the Kobayashi-Maskawa Matrix, Phys. Rev. Lett. 53, 1802 (1984). 